\title{
The nature of the serum alkaline phosphatases in liver diseases
}

\author{
C. P. PRICE AND H. G. SAMMONS \\ From the Department of Clinical Chemistry, East Birmingham Hospital, Birmingham
}

SYNOPSIS The two main alkaline phosphatase isoenzymes in serum from patients with extrahepatic biliary obstruction have been purified. Properties of these isoenzymes, such as electrophoretic mobility, separation on gel filtration, ultracentrifugation characteristics, Michaelis constants, and sensitivity to neuraminidase have been studied and compared with the isoenzymes of liver and bile. The results show that there is an abnormal isoenzyme in the serum from these patients and that this isoenzyme is similar but not identical with the main bile isoenzyme. It is suggested that it may be associated with a lipoprotein carrier.

The estimation of serum alkaline phosphatase has been employed as a liver function test for many years although its value has often been questioned. Its specificity has, however, been improved as a result of techniques available for the differentiation of alkaline phosphatase isoenzymes.

Electrophoretic studies of serum alkaline phosphatase isoenzymes from patients with obstructive liver disease have indicated the presence of an abnormal isoenzyme that differs from that found in liver tissue (Hodson, Latner, and Raine, 1962; Chiandussi, Green, and Sherlock, 1962; Gordon, 1965; Hill and Sammons, 1967; Wieme and Demeulenaere, 1971).

The present study was undertaken to determine the nature of the serum alkaline phosphatase isoenzymes in patients with obstructive jaundice in relation to the work on bile alkaline phosphatase previously described (Price, Hill, and Sammons, 1972).

\section{Materials and Methods}

Sera were obtained from 58 patients with extrahepatic biliary obstruction diagnosed at laparotomy or necropsy, and from 20 laboratory staff who acted as normal controls. Specimens of hepatic bile were obtained from 12 patients with T-tube drainage of the common bile duct after cholecystectomy. Samples of liver and intestine were obtained from necropsy material; crude homogenates were made from these samples for use as tissue controls. Serum

Received for publication 5 March 1974. was obtained from a patient with Paget's disease for use as a bone alkaline phosphatase control.

Electrophoretic studies were performed in polyacrylamide gel discs using the Shandon electrophoresis equipment. The procedure was similar to that of Smith, Lightstone, and Perry (1968), modified to include a $3.5 \%(\mathrm{w} / \mathrm{v})$ polyacrylamide 'topping gel' above the $5.0 \%(\mathrm{w} / \mathrm{v})$ running gel. This topping gel was employed so that the isoenzyme that remained at the origin could be separated from a nonspecific staining reaction at the top of the gel. This isoenzyme was able to penetrate the $3.5 \%$ $(w / v)$ gel.

Cellulose acetate electrophoresis was employed using the Sepratek system (Gelman Instruments Limited, Michigan, USA) and a barbitone buffer pH 8.6 (Kohn and Feinberg, 1965).

Visualization of the alkaline phosphatase isoenzymes was attained by means of incubation with $\beta$-napthyl phosphate and Fast Blue RR according to Smith et al (1968). In the case of the cellulose acetate technique the strips were incubated with substrate only and the reaction products visualized separately, to avoid heavy background staining. Quantitative localization of enzyme activity after disc electrophoresis was accomplished by cutting the gels into $0.5 \mathrm{~cm}$ segments and estimating the activity in the fraction homogenized in buffered substrate by the method of Kind and King (1954); $10 \mathrm{mM} \mathrm{Mg}{ }^{++}$was included in the reaction mixture.

Gel filtration studies were carried out with Sephadex G200 (Pharmacia, Uppsala, Sweden). The gel was prepared and equilibrated in a $2 \times 60 \mathrm{~cm}$ column with $0.01 \mathrm{M}$ Tris- $\mathrm{HCl} \mathrm{pH} 7.4$ buffer containing 1.0 392 
$M$ sodium chloride. Samples $(2.0 \mathrm{ml})$ were applied to the base of the column and the proteins fractionated by upward displacement at a flow rate of $5 \mathrm{ml}$ per hour. The eluate was scanned at $280 \mathrm{~nm}$ using a Uvicord II absorptiometer (LKB Produkter, Sweden) and fractions were collected over 20-minute periods and assayed for alkaline phosphatase activity.

Ultracentrifugation studies were carried out by a method similar to that of Pope and Cooperband (1966). A gradient ranging from 10 to $40 \%$ (w/v) sucrose in distilled water was constructed in the Spinco SW50 swinging bucket head and $0.5 \mathrm{ml}$ of sample layered on top. The tubes were then spun for 18 hours at $32000 \mathrm{rpm}$. The tubes were fractionated by upward displacement with $50 \%$ (w/v) sucrose. The absorption at $280 \mathrm{~nm}$ was monitored and $15 \times 0.3$ $\mathrm{ml}$ fractions were collected. The positions of the 7S and 19S proteins were detected by assaying the levels of IgG and IgM respectively in each fraction of a serum control sample. Alkaline phosphatase activity was determined on each fraction.

To purify the alkaline phosphatase isoenzymes, $10 \mathrm{ml}$ aliquots of serum (mean activity 50 KindKing units per $100 \mathrm{ml}$ ) were first treated with dextran sulphate according to the method of Stokes (1965). To the supernatant was added anhydrous sodium sulphate to a concentration of $17 \%(w / v)$. The resulting precipitate was separated by centrifugation. The concentration of sodium sulphate was increased in $2 \%(\mathrm{w} / \mathrm{v})$ stages by the addition of more anhydrous sodium sulphate, removing the resulting precipitate at each stage by centrifugation. Alkaline phosphatase activity was found in two fractions: one in the precipitate between 19 and $25 \%$ sodium sulphate and the second in the supernatant at $25 \%$. The precipitates containing activity were redissolved in $0.1 \mathrm{M}$ Tris- $\mathrm{HCl}$ buffer $\mathrm{pH} 7.4$ and both alkaline phosphatase fractions concentrated by reduced pressure ultrafiltration in Visking tubing at $4^{\circ} \mathrm{C}$. Each fraction was separately purified by gel filtration as described above and by ion exchange chromatography (Price et al, 1972).

In an effort to characterize the isoenzyme fractions further, the effect of neuraminidase was investigated (Hill and Sammons, 1967). The Michaelis constants of the two fractions were also obtained and compared with that of bile isoenzymes, at a constant $\mathrm{pH} 9.8$ over the range $0-5 \mathrm{mM}$ disodium phenyl phosphate.

The effect of butanol extraction on the electrophoretic mobility of the isoenzyme fractions was assessed using polyacrylamide gel electrophoresis. Because of the reported findings with the bile alkaline phosphatases, thin-layer chromatographic procedures (Price et al, 1972) were carried out on the butanol extract from each isoenzyme fraction to identify any lipid components present.
Results

\section{ELECTROPHORESIS}

The serum alkaline phosphatase isoenzyme patterns were studied in 58 patients with extrahepatic biliary obstruction (fig 1). The main serum alkaline phosphatase isoenzyme migrated in polyacrylamide gel to a position equivalent to the liver tissue isoenzyme and in front of the bone isoenzyme. A further isoenzyme was found to be present at the origin, contributing at least $20 \%$ to the total activity. Three further isoenzyme bands were detected in much smaller quantities, contributing to less than $10 \%$ of the total activity. Two patients ( $g$ ) showed a faint band of activity that migrated in a position equivalent to the intestinal alkaline phosphatase. Four patients (e) showed a faint band of activity that migrated a short distance into the $5 \%$ gel, and four patients (f) showed a faint band of activity at the junction of the 3.5 and $5 \%$ gels. This band could be abolished by heating so was assumed not to be an artefact. The presence of these minor bands bore no relation to enzyme activity or type of biliary obstruction. As previously found, the main bile isoenzyme remained at the origin (Price et al, 1972).

The main serum isoenzyme on cellulose acetate electrophoresis was in the $\alpha_{2}$-globulin region with a

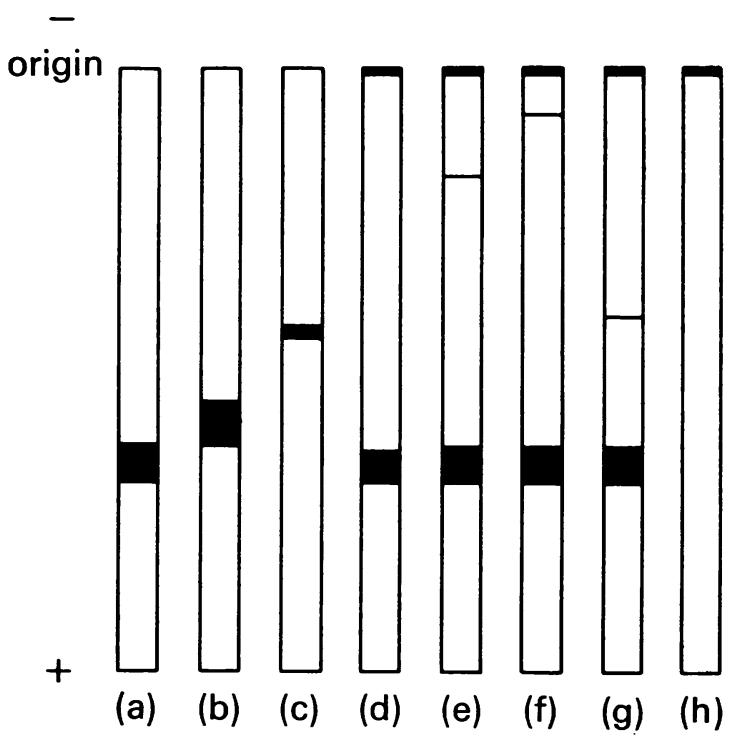

Fig 1 Separation of serum alkaline phosphatase isoenzymes in patients with extrahepatic biliary obstruction following polyacrylamide gel electrophoresis: (a) liver tissue preparation; (b) serum from patients with Paget's disease; (c) intestinal tissue preparation; (d) 48 patients; (e) four patients; (f) four patients; (g) two patients; (h) hepatic bile. 


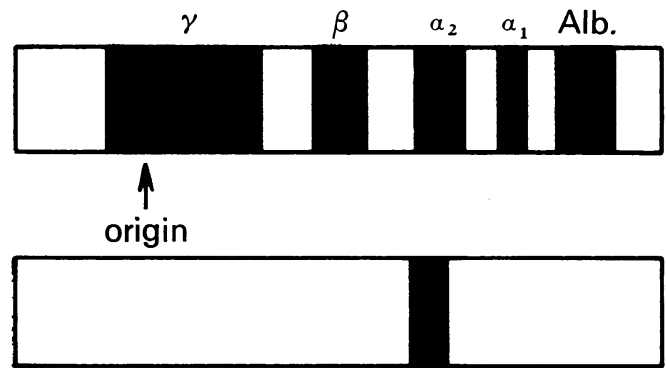

(b)

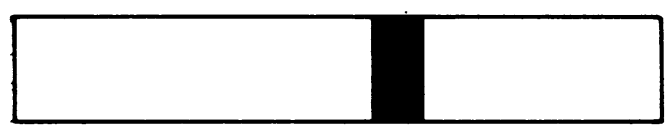

(c)

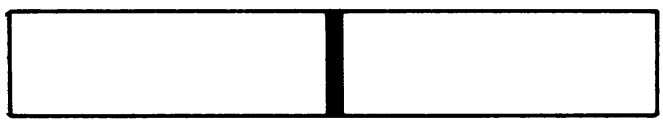

(d)
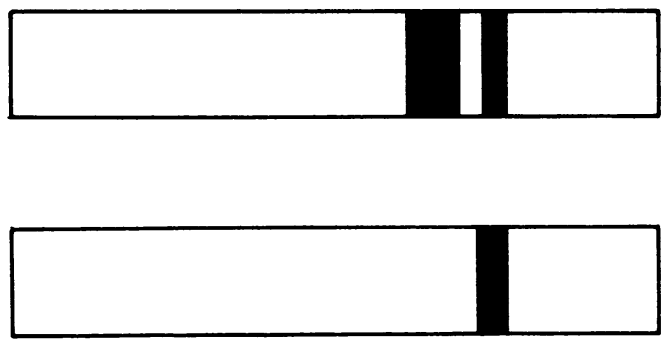

Fig 2 Serum alkaline phosphatase isoenzymes after cellulose acetate electrophoresis: (a) serum proteins; (b) liver tissue preparation; (c) serum from patients with Paget's disease; (d) intestinal tissue preparation;

(e) patients with extrahepatic obstruction; (f) hepatic bile.

less prominent band in the $\alpha_{1}$-globulin region (fig 2 ). This $\alpha_{1}$-globulin band was found to be equivalent to the 'origin' band on disc electrophoresis. The bile samples also showed one major band of activity in the $\alpha_{1}$-globulin region.

GEL FILTRATION

Gel filtration fractionation of serum proteins showed three main peaks (fig 3), as described by Flodin and Killander (1962). In 20 normal people alkaline phosphatase activity was observed in the second (7S) protein peak. Studies of the control samples indicated that the liver and bone alkaline phosphatases were found solely in the $7 \mathrm{~S}$ protein fraction. The intestinal
Fig 3 Gel filtration characteristics of various alkaline phosphatase isoenzymes:

(a)

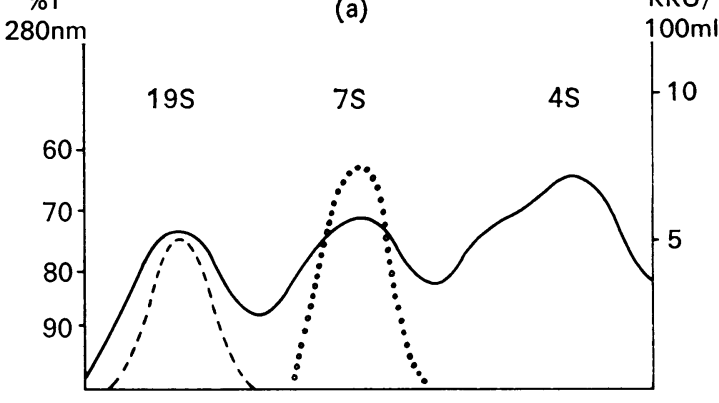

(b)

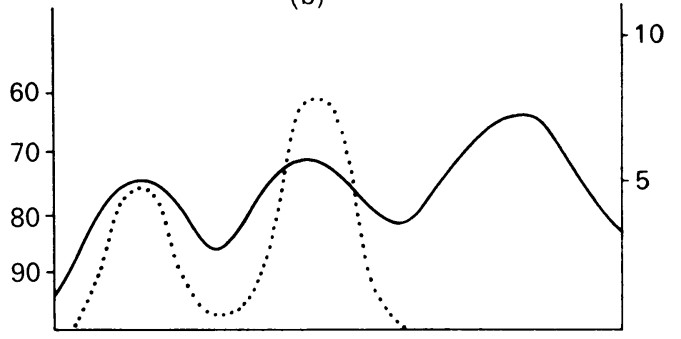

(a) - liver tissue ---- Serum proteins

(e) bile; (b) .......... sera from patients with extrahepatic obstruction.

alkaline phosphatase was found in the 7S peak with a shoulder on the leading edge of the peak.

In eight samples of hepatic bile the main fraction (f) of alkaline phosphatase was found in the first protein fraction; this was found to correspond with the 'origin' isoenzyme on electrophoresis. Where the bile showed a proportion of 'liver' alkaline phosphatase a second fraction was found on gel filtration in the $7 \mathrm{~S}$ protein fraction.

Sera from patients with extrahepatic obstruction showed two peaks of alkaline phosphatase activity equivalent to the $19 \mathrm{~S}$ and $7 \mathrm{~S}$ protein fractions (fig $3 b)$. In the eight samples studied there was a good o relationship between the contribution of the 'origin' isoenzymes on electrophoresis and the 19S fraction, which ranged between 12 and $42 \%$ of the total activity (table I).

ULTRA CENTRIFUGATION

Ultracentrifugation of four samples of hepatic bile was performed. Only one fraction of alkaline phosphatase activity was found, in the position of the 7S proteins. In 18 samples of sera from patients with extrahepatic obstruction two fractions of alkaline phosphatase activity were observed, one in the $7 \mathrm{~S}$ protein fraction and one floating at the top of the 


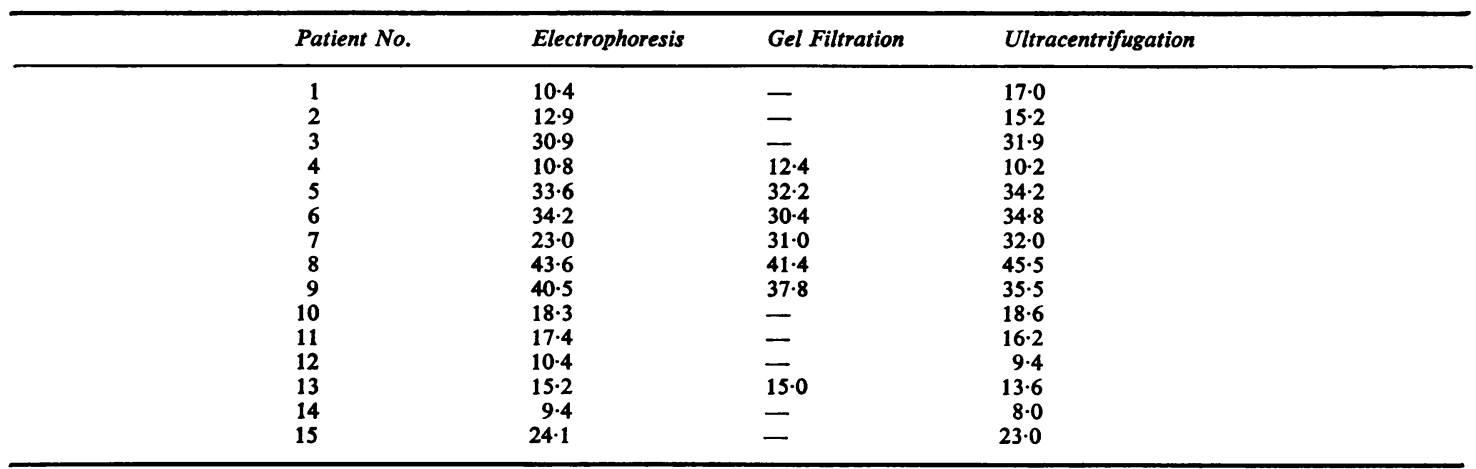

Table I Comparison of quantitative results for the origin isoenzyme on electrophoresis, $19 S$ fraction on gel filtration, and upper fraction on ultracentrifugation, of sera from patients with hepatobiliary disorders ${ }^{1}$

${ }^{1}$ The enzyme activity has been expressed as a percentage of the total serum activity.

sucrose gradient. The floating fraction after ultracentrifugation on electrophoresis was found to correspond with the 'origin' isoenzymes previously observed (table I). Quantitative distribution showed that this fraction contributed $12-15 \%$ to the total alkaline phosphatase activity (fig 4).

COMPARISON OF SEPARATION TECHNIQUES Several samples were studied by the electrophoretic technique with quantitative localization, the gel

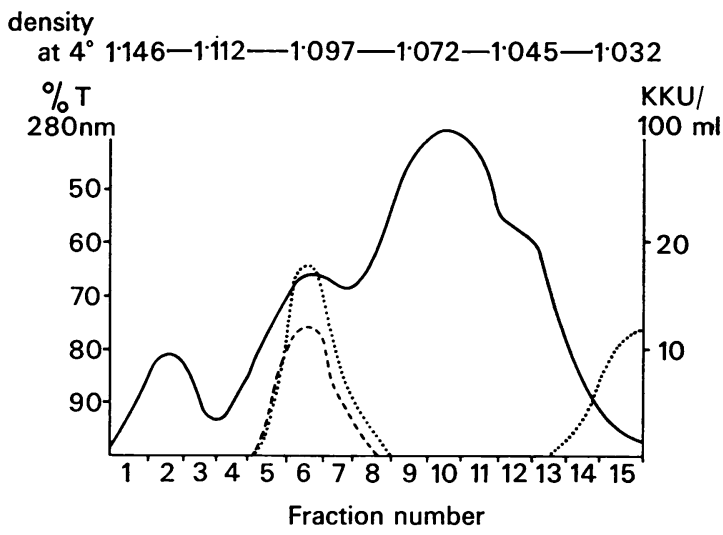

Fig 4 Ultracentrifugation characteristics of serum and bile alkaline phosphatase isoenzymes: serum proteins, - - - - - hepatic bile, ......... serum from patients with extrahepatic obstruction. filtration and ultracentrifugation techniques. The quantitative distribution of the main isoenzyme fractions is compared in table I and shows that there is close agreement between the estimates of the abnormal serum isoenzyme activity separated by the three techniques described.

\section{PURIFICATION AND PROPERTIES OF}

ISOENZYMES

In view of the association of the bile alkaline phosphatase isoenzymes with a lipid component (Price et al, 1972) attempts to purify the serum isoenzymes initially involved a delipidation stage. It was found that treatment of serum with dextran sulphate resulted in no loss of activity or change in electrophoretic mobility. This technique was followed by salt fractionation and ion exchange and gel filtration chromatography. These procedures resulted in a $56 \%$ recovery of enzyme with a 123 -fold increase in specific activity of the 'origin' isoenzyme and a 162fold increase in specific activity of the 'liver' isoenzyme.

Ultracentrifugation and electrophoretic studies were carried out at each stage of the purification; after sodium sulphate precipitation these studies were confined to the fraction precipitating between 19 and $25 \%$ sodium sulphate. The results shown in table II indicate a change in the density of the molecule without change in mobility after DEAE cellulose chromatography.

\begin{tabular}{lll}
\hline Stage of Purification & Electrophoretic Mobility & Ultracentrifugation Characteristics \\
\hline After sodium sulphate $19-25 \%$ & 'Origin' & Top of gradient \\
After DEAE cellulose chromatography & 'Origin' & 7S Fraction \\
After gel filtration & 'Origin' & 7S Fraction \\
\hline
\end{tabular}

Table II Electrophoretic mobility and ultracentrifugation characteristics of the serum alkaline phosphatase isoenzyme found at the origin after electrophoresis 
Extraction of the purified isoenzyme preparations with butanol caused no change in the liver isoenzyme. There was no change in activity of the 'origin' isoenzymes but a change in mobility was noted to a position equivalent to that of the liver isoenzyme. Extraction of the bile 'origin' isoenzyme, as previously reported, resulted in not only a similar change in electrophoretic mobility but also an overall increase in activity.

Incubation with neuraminidase caused a decrease in the electrophoretic mobility of the faster serum isoenzyme; this corresponded to the result found using the liver tissue alkaline phosphatase. The mobility of the alkaline phosphatase remaining at the origin was not altered by neuraminidase. However, when this origin fraction was extracted with butanol and then incubated with neuraminidase it showed a decrease in mobility identical to that of the liver alkaline phosphatase. These results are shown in table III and are expressed as the ratio of the distance moved by the test (incubated with neuraminidase) over the distance moved by the control (incubated with inactivated neuraminidase). Treatment of the 'bone' alkaline phosphatase from the serum of a patient with Paget's disease showed a much greater decrease in electrophoretic mobility.

The apparent $\mathrm{Km}$ values were calculated at a $\mathrm{pH}$ of 9.8. The results for various isoenzyme fractions are shown in table IV. These data represent the mean of results on at least six isoenzyme preparations in each case. The $\mathrm{Km}$ values were determined from Lineweaver Burke plots by the method of least squares regression. It can be seen that the $\mathrm{Km}$ of the faster bands of serum and bile and the 'origin' isoenzyme of serum differ markedly from the 'origin' isoenzyme of bile. Butanol extraction of the bile 'origin' isoenzyme resulted in a decrease in $\mathrm{Km}$ value but there was no change in the $\mathrm{Km}$ value of the extracted serum 'origin' isoenzyme.

The thin-layer chromatography carried out on the butanol extracts of the two serum isoenzyme preparations indicated the presence of a phospholipid in the 'origin' isoenzyme extraction solvent. Further \& chromatography in a second solvent system showed this component to be a phosphatidylcholine. The other isoenzyme solvent extract yielded no detectable lipid component. In order to ascertain whether the phosphatidylcholine was a normal finding, a sample of normal serum was fractionated in a similar manner to the abnormal samples and the resulting preparation, containing no alkaline phosphatase, was extracted with butanol. The solvent phase did not contain any detectable lipid component.

\section{Discussion}

Few attempts have been made to correlate the data obtained by different techniques for the differentiation of serum alkaline phosphatase isoenzymes in liver disease. Our results using cellulose acetate electrophoresis show that in obstructive liver disease the main enzyme activity is found in the $\alpha_{2}$-globulin fraction with lesser activity in the $\alpha_{1}$-globulin fraction; the latter fraction is not found in normal control serum. These results are in agreement with previous results employing electrophoretic techniques relying mainly on the net charge on the protein molecule, and using, for example, paper (Baker and

\begin{tabular}{|c|c|c|c|}
\hline \multirow[t]{2}{*}{ Samples } & \multicolumn{3}{|c|}{ Mobility $(\mathrm{cm})$} \\
\hline & Control & Test & Ratio $T / C$ \\
\hline $\begin{array}{l}\text { Main serum isoenzyme } \\
\text { 'Origin' serum isoenzyme } \\
\text { Liver tissue isoenzyme } \\
\text { Serum }\end{array}$ & $\begin{array}{l}9 \cdot 4 \\
0 \cdot 1 \\
9 \cdot 7\end{array}$ & $\begin{array}{l}6 \cdot 9 \\
0 \cdot 1 \\
7 \cdot 2\end{array}$ & $\begin{array}{l}0.74 \\
1.00 \\
0.74\end{array}$ \\
\hline $\begin{array}{l}\text { Paget's disease isoenzyme } \\
\text { 'Origin' isoenzyme after butanol treatment }\end{array}$ & $\begin{array}{l}9 \cdot 5 \\
9 \cdot 4\end{array}$ & $\begin{array}{l}5 \cdot 0 \\
6 \cdot 8\end{array}$ & $\begin{array}{l}0.53 \\
0.72\end{array}$ \\
\hline
\end{tabular}

Table III Effect of neuraminidase on the electrophoretic mobility of various alkaline phosphatase isoenzymes

\begin{tabular}{|c|c|c|c|c|}
\hline & \multicolumn{4}{|c|}{ Mean Apparent $\mathrm{Km}$ Value (mM) } \\
\hline & \multicolumn{2}{|l|}{ Bile } & \multicolumn{2}{|l|}{ Serum } \\
\hline & 'Fast' Band & 'Origin' Band & 'Fast' Band & 'Origin' Band \\
\hline $\begin{array}{l}\text { Constant pH } 9.8 \\
\text { Constant pH } 9.8 \text { after butanol extraction }\end{array}$ & $\begin{array}{l}2.04 \\
2.02\end{array}$ & $\begin{array}{l}2 \cdot 63 \\
2.04\end{array}$ & $\begin{array}{l}2.00 \\
2.02\end{array}$ & $\begin{array}{l}1.94 \\
1.94\end{array}$ \\
\hline
\end{tabular}

Table IV Apparent Km values of purified bile and serum alkaline phosphatase isoenzymes determined at a constant $p H$ 
Pellegrino, 1954), starch block (Rosenberg, 1959; Keiding, 1969), cellulose acetate (Korner, 1962), and agar gel (Wieme and Demeulenaere, 1971).

Using polyacrylamide gel electrophoresis we found that in patients with obstructive liver disease the serum alkaline phosphatase showed a gross increase in the fast liver isoenzyme fraction with the appearance of several other isoenzyme fractions, the major contribution remaining at the origin. Our results are in agreement with those of other workers who employed similar techniques which included molecular sieving superimposed on the true electrophoretic mobility, for example, starch gel (Kowlessar, Pert, Haeffner, and Sleisenger, 1959; Moss, Campbell, Anagnostou-Karakas, and King, 1961; Chiandussi et al, 1962; Hodson et al, 1962; Taswell and Jeffers, 1963; and Hill and Sammons, 1967) and polyacrylamide gel (Jennings, Brocklehurst, and Hirst, 1970; Walker and Pollard, 1971).

Our studies suggest that the isoenzyme migrating with the 'liver' fraction on polyacrylamide gel is equivalent to the $\beta$-globulin fraction on starch gel and the $\alpha_{2}$-globulin fraction on cellulose acetate. Furthermore, the results suggest that the isoenzyme remaining at the origin on acrylamide gel is identical with the 'origin' and $\alpha_{1}$-globulin fractions of starch gel and cellulose acetate respectively. The main bile isoenzyme on cellulose acetate electrophoresis was found in the $\alpha_{1}$-globulin fraction and at the origin using polyacrylamide gel electrophoresis. We conclude from these findings that the abnormal serum isoenzyme differs significantly from the main 'liver' alkaline phosphatase isoenzyme not only in terms of net charge but also in terms of size. However, it appears to behave similarly with respect to electrophoretic mobility to the main bile isoenzyme.

Using gel filtration, the liver tissue isoenzyme as well as the bone and intestinal isoenzymes were found in the 7S protein fraction. In the majority of bile samples the alkaline phosphatase activity was found mainly in the $19 \mathrm{~S}$ protein fraction although a second fraction was occasionally found with the 7S proteins. When all of the alkaline phosphatase activity was found in the $19 \mathrm{~S}$ protein fraction it was observed that only the 'origin' isoenzyme was found after polyacrylamide gel electrophoresis; the 7S protein fraction containing enzyme activity had the mobility of 'liver' alkaline phosphatase on electrophoresis.

Our gel filtration studies of sera from patients with liver diseases confirmed those of previous workers by showing that in general there are two alkaline phosphatase isoenzyme fractions, the major peak of activity being present in the $7 \mathrm{~S}$ protein fraction with small amounts in the 19S fraction (Dunne, Fennelly, and McGeeney, 1967; Jennings et al, 1970). On polyacrylamide gel, electrophoresis of concentrated 7S and 19S alkaline phosphatase fractions showed electrophoretic mobility identical with the 'liver' and 'origin' isoenzymes. It can be seen from table I that the contributions of the 'origin' isoenzyme by quantitative localization following electrophoresis agreed well with the contributions of the 19S alkaline phosphatase fractions to the total activity.

The present work confirms the findings of Pope and Cooperband (1966) who, using an ultracentrifugation technique, observed that the bile alkaline phosphatase isoenzymes and normal serum isoenzyme float with the 7S proteins. These authors further suggested that the abnormal serum isoenzyme was also found with the $7 \mathrm{~S}$ proteins. Our results, however, show that the abnormal serum isoenzyme has a much lower density than the 'liver' isoenzyme as it was found to float to the top of the sucrose gradient. The quantitative distribution studies, shown in table I, indicate that the contribution of this fraction to the total activity is very similar to that of the 'origin' isoenzyme on electrophoresis.

Hill (1967) first reported that extraction of bile with $\mathrm{n}$-butanol resulted in an overall increase in alkaline phosphatase activity. This was accompanied by a change in electrophoretic mobility of the 'origin' isoenzyme to the $\beta$-globulin position on starch gel. It was subsequently found that extraction of sera from patients with liver disease did not result in an overall increase in enzyme activity but did result in a change in electrophoretic mobility of one isoenzyme from the origin to the $\beta$-globulin position. Jennings et al (1970) and Walker and Pollard (1971) also found that butanol extraction of an abnormal serum isoenzyme resulted in a change in its electrophoretic mobility. No form of quantitation was, however, included in these studies.

The work presented shows that there are many similarities between the main bile isoenzyme and the abnormal serum 'origin' isoenzyme. However, certain fundamental differences do exist which are apparent from the ultracentrifugation data. Results show that the abnormal serum 'origin' isoenzyme is less dense than the main bile isoenzyme (fig 4) but the density increases during purification by ion exchange chromatography (table II). Butanol extraction of the two slow moving isoenzymes results in identical changes in electrophoretic mobility, the resulting isoenzymes being identical in every respect studied. However, there appears to be a decrease in the $\mathrm{Km}$ value at $\mathrm{pH} 9.8$ of the bile isoenzyme to a value similar to that of the serum 'origin' isoenzyme, which is unaltered by extraction.

The changes in ultracentrifugation characteristics 
during purification suggest that a 'light' component is split from the enzyme molecule during ion exchange chromatography. The enzyme molecule so produced is then virtually identical with the main bile isoenzyme in its electrophoretic mobility and behaviour with butanol. The differences in $\mathrm{Km}$ value of the two 'origin' isoenzymes may reflect the combination of the serum isoenzyme with another component, possibly lipoprotein.

There are several possible theories to explain the presence of this abnormal isoenzyme in the serum. In the first place, this isoenzyme could represent a polymerization of the normal isoenzyme, but this would seem unlikely in view of the low buoyant density of the molecule relative to the normal enzyme. By the same reasoning it is unlikely that the abnormal enzyme reflects linkage of the normal enzyme to another protein. Secondly, the possibility of an enzyme-carbohydrate complex is probably ruled out by the neuraminidase experiments and the insensitivity of the isoenzyme to this treatment.

The concept of lipoprotein carriage of an enzyme has been suggested by many workers (Moss, 1962; Latner, 1966; Dunne et al, 1967; Jennings et al, 1970). The low buoyant density of the abnormal serum isoenzyme would support the idea of lipoprotein carriage; ion exchange chromatography may remove this carrier. This is the first time that the nature of the carried enzyme has been suggested. Our work has shown that a lipid component has been extracted from the purified abnormal serum isoenzyme which is identical to the lipid component of the main bile isoenzyme. Furthermore, the extracted serum isoenzyme shows identical characteristics to the extracted bile isoenzyme.

Taking all these facts into account, therefore, our results indicate that in obstructive liver disease the following situation exists with respect to the raised serum alkaline phosphatase activity. There are two main isoenzymes present: one is an $\alpha_{2}$-globulin derived directly from the liver cells, which contributes the major fraction. The second isoenzyme is present in smaller proportions and represents the regurgitation of bile alkaline phosphatase as a result of obstruction to the normal flow of bile. The abnormal serum isoenzyme probably represents the 'bile' isoenzyme, a 'lecithin-liver isoenzyme complex' (Price et al, 1972) associated with a lipoprotein carrier thus increasing its effective size and affecting its electrophoretic mobility in certain media. The source, site, and method of addition of this lipoprotein carrier remain unknown. These observations have nevertheless allowed a pattern of isoenzyme changes to be determined and followed in liver diseases. These results will be the subject of a further communication.

References

Baker, R. W. R., and Pellegrino, C. (1954). The separation and detection of serum enzymes by paper electrophoresis. Scand. J. clin. Lab. Invest., 6, 94-101.

Chiandussi, L., Greene, S. F., and Sherlock, S. (1962). Serum alkaline phosphatase fractions in hepatobiliary and bone diseases. Clin. Sci., 22, 425-434.

Dunne, J., Fennelly, J. J., and McGeeney, K. (1967). Separation of ญ alkaline phosphatase isoenzymes in human serum using gelfiltration (Sephadex G200) techniques. Cancer (Philad.), 20, $\overrightarrow{0}$ $71-76$.

Flodin, P., and Killander, J. (1962). Fractionation of human serum $\overrightarrow{\vec{A}}$ proteins by gel filtration. Biochim. biophys. Acta (Amster.), $\omega$ 63, 403-410.

Gordon, S. (1965). Alkaline phosphatase isoenzymes in serum and $\frac{\widehat{O}}{0}$

tissues. $S$. Afr. med. J., 39, 49-53.
Hill, P. G. (1967). Studies on alkaline phosphatase and $5^{\prime}$-nucleotidase $\mathcal{N}$ in human subjects in health and disease. PhD Thesis, University i of Birmingham.

Hill, P. G., and Sammons, H. G. (1967). An interpretation of the elevation of serum alkaline phosphatase in disease. J. clin. $\mathrm{N}$ Path., 20, 654-659.

Hodson, A. W., Latner, A. L., and Raine, L. (1962). Isoenzymes of alkaline phosphatase. Clin. chim. Acta, 7, 255-261.

Jennings, R. C., Brocklehurst, D., and Hirst, M. (1970). A com- $\$ parative study of alkaline phosphatase enzymes using starch gel electrophoresis and Sephadex gel filtration with special reference to high molecular weight enzymes. Clin. chim. Acta, $\overrightarrow{0}$ 30, 509-517.

Keiding, R. (1969). Mechanism of phosphohydrolase transport and : homeostasis. Ann. N.Y. Acad. Sci., 166, 510-522.

Kind, P. R. N., and King, E. J. (1954). Estimation of plasma phosphatase by determination of hydrolysed phenol with aminoantipyrine. J. clin. Path., 7, 322-326.

Kohn, J., and Feinberg, J. G. (1965). Electrophoresis on cellulose acetate. Shandon Instruments Applications No. 11, Shandon Scientific Company, London.

Korner, N. H. (1962). Distribution of alkaline phosphatase in serum protein fractions. J. clin. Path., 15, 195-199.

Kowlessar, O. D., Pert, J. H., Haeffner, L. J., and Sleisenger, M. H. (1959). Localization of 5 -nucleotidase and non-specific $\frac{-}{T}$ alkaline phosphatase by starch gel electrophoresis. Proc. Soc. exp. Biol. (N.Y.), 100, 191-196.

Latner, A. L. (1966). In Transport Function of Plasma Proteins, edited by P. Desgrez and P. M. De Traverse, p. 122. Elsevier, $\frac{O}{O}$ Amsterdam.

Moss, D. W., Campbell, D. M., Anagnostou-Karakas, E., and King, E. J. (1961). Characterization of tissue alkaline phosphatases. and their partial purification by starch-gel electrophoresis. Biochem. J., 81, 441-447.

Moss, D. W. (1962). Isoenzymes of alkaline phosphatase in autolyzed and butanol extracted liver preparations. Nature (Lond.), 193, 음 981-982.

Pope, C. E., and Cooperband, S. R. (1966). Protein characteristics of $\frac{D}{O}$ serum and bile alkaline phosphatase. Gastroenterology, 50, 을. 631-636.

Price, C. P., Hill, P. G., and Sammons, H. G. (1972). The nature of the alkaline phosphatases of bile. J. clin. Path., 25, 149-154.

Rosenberg, I. E. (1959). Zone electrophoretic studies of serum $\mathrm{N}$ alkaline phosphatase. J. clin. Invest., 38, 630-644.

Smith, I., Lightstone, P. J., and Perry, J. D. (1968). Separation of human tissue alkaline phosphatases by electrophoresis on
acrylamide disc gels. Clin. chim. Acta, 19, 499-505.

Stokes, R. P. (1966). A quantitative small scale method for the separation of low density (beta) lipoproteins from serum. $\frac{\mathrm{C}}{\mathbb{D}}$ J. med. Lab. Technol., 23, 33-34.

Taswell, H. F., and Jeffers, D. M. (1963). Isoenzymes of serum: alkaline phosphatase in hepatobiliary and skeletal disease. Amer. J. clin. Path., 40, 349-356.

Walker, A. W., and Pollard, A. C. (1971). Observations on serum $\frac{\vec{D}}{\vec{D}}$ alkaline phosphatase electrophoretic patterns on polyacrylamide gel. Clin. chim. Acta, 34, 19-29.

Wieme, R. J., and Demeulenaere, L. (1971). Enzyme assays in liver disease. J. clin. Path., 24, Suppl. (Ass. Clin. Path.), 4, 51-59. 